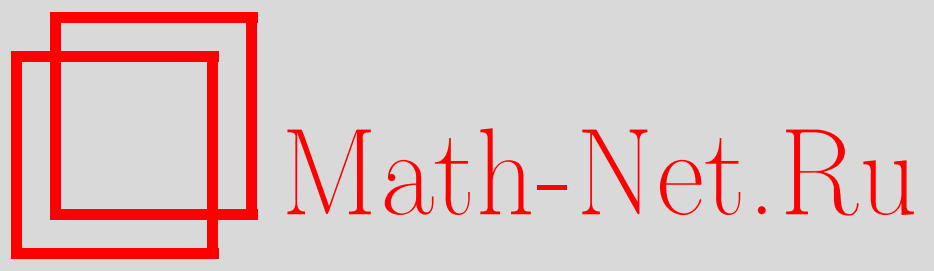

С. А. Вакуленко, М. В. Черкай, Разрушение диссипативных структур при случайных воздействиях, ТMФ, 2010, том 165, номер 1, 177-192

DOI: https://doi.org/10.4213/tmf6570

Использование Общероссийского математического портала Math-Net.Ru подразумевает, что вы прочитали и согласны с пользовательским соглашением http://www.mathnet.ru/rus/agreement

Параметры загрузки:

IP : 3.89.197.203

26 апреля 2023 г., 04:24:24

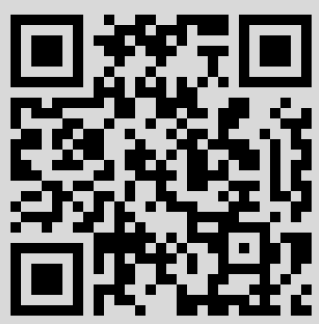




\title{
РАЗРУШЕНИЕ ДИССИПАТИВНЫХ СТРУКТУР ПРИ СЛУЧАЙНЫХ ВОЗДЕЙСТВИЯХ
}

\begin{abstract}
Рассматриваются системы химической кинетики со случайными параметрами, важные во многих физических, химических и биологических приложениях. Случайные параметры описывают действие флуктуаций внешней среды на систему. Даны оценки вероятности того, что состояние системы на интервале времени $[0, T]$ остается в некоторой заданной области фазового пространства при условии, что оно было в начальный момент в $u_{0}$, где $u_{0}$ - равновесное решение, описывающее диссипативную структуру. Показано, что задача максимизации этой вероятности в некоторых случаях может быть сведена к известной задаче о минимизации гамильтониана Хопфилда.
\end{abstract}

Ключевые слова: системы реакции-диффузии, случайные параметры, гомеостазис, диссипативные структуры, спиновые стекла.

\section{1. ВВЕДЕНИЕ}

В настоящей работе рассматриваются системы химической кинетики со случайными параметрами, важные во многих физических, химических и биологических приложениях. Решения этих систем могут описывать стационарные пространственно неоднородные структуры (диссипативные структуры), периодические колебания и волны, хаос. Важно учесть влияние флуктуаций внешней среды на эти структуры. В частности, мы рассматриваем гипотезу, предложенную Громовым и Карбоне [1]: "Гомеостазис индивидуальной клетки не может быть устойчивым долгое время, так как он будет разрушен случайными флуктуациями внутри и вне клетки. Не существует адекватного математического формализма, который бы выразил интуитивно ясную идею репликативной устойчивости динамических систем".

Математическая формализация понятия гомеостазиса может быть сделана в рамках так называемой теории выживания [2] и идей, изложенных в монографии [3].

Рассматриваются глобальные полупотоки в фазовом пространстве $H$ со случайными параметрами, зависящими от времени. Эти полупотоки заданы уравнениями в

${ }^{*}$ Санкт-Петербургский государственный университет технологии и дизайна, СанктПетербург, Россия. E-mail: vakulenfr@mail.ru

${ }^{\dagger}$ Институт проблем машиноведения РАН, Санкт-Петербург, Россия. E-mail: chmv2007@rambler.ru 
частных производных типа систем химической кинетики со случайными параметрами, зависящими от времени. Слагаемые со случайными параметрами входят в правые части дифференциальных уравнений аддитивно. В пространстве $H$ выделяется некоторое подмножество П, содержащее аттрактор полупотока $\mathcal{A}$. Предполагается, что если состояние системы покидает подмножество П, то система разрушается.

В данной статье мы рассматриваем простейшую ситуацию, когда аттрактор представляет собой точку $u_{0} \in H$ - стационарное решение, которое может описывать как пространственно однородное, так и неоднородное решение (диссипативную структуру). Считается, что гомеостазис поддерживается и система не разрушается, если состояние $u \in \Pi$. Мы даем оценки вероятности $P\left(T, \Pi, u_{0}\right)$ того, что состояние системы остается в области П на интервале времени $[0, T]$, при условии, что оно было в начальный момент в точке $u_{0}$.

Сначала мы доказываем, что для почти всех (в смысле дифференциальной топологии) систем химической кинетики имеем $P\left(T, \Pi, u_{0}\right) \rightarrow 0$ при $T \rightarrow \infty$. Таким образом, для данного важного класса систем гипотеза Громова-Карбоне может быть подтверждена в ее негативной части. Здесь существенно, что допускаются сколь угодно большие значения случайных параметров.

Эксперименты показывают, что многие реальные биологические диссипативные структуры не разрушаются даже при относительно больших вариациях внешних и внутренних параметров. Например, при вариациях размера $L$ системы порядка $0.2 L$ периодическая слоистая структура сохраняется и число слоев не изменяется [4], что на первый взгляд противоречит полученному результату. Это может быть объяснено тем, что вероятность $Q=1-P$ разрушения структуры может быть мала. Показывается, что она мала именно для типичных во многих приложениях ситуаций, когда имеются компоненты, диффундирующие быстро и диффундирующие медленно.

Далее мы исследуем вероятности разрушения структуры в зависимости от ее свойств и числа слоев в ней. Мы рассматриваем задачу о нахождении структур, имеющих, в некотором смысле, достаточно малые вероятности разрушения. Если допустить, что уравнения зависят от некоторого дополнительного дискретного параметра, то оказывается, что эта задача оптимизации связана с вопросами, возникающими в дискретной математике и теории нейронных сетей, которые были в центре внимания многих физиков-теоретиков и математиков последние годы [5]-[8]. Она сводится в простейшем случае к минимизации энергии спинового стекла, определенной гамильтонианом Хопфилда. При некоторых естественных предположениях показано, что возможны структуры $u_{0}$, вероятность разрушения которых мала: $P\left(T, \Pi, u_{0}\right)>1-\nu(N), \nu \rightarrow 0$ при $N \rightarrow \infty$, где $N$ - число слоев в структуре. Дается описание алгоритма поиска такой структуры.

\section{2. ОБЩАЯ МАТЕМАТИЧЕСКАЯ ФОРМУЛИРОВКА ПРОБЛЕМЫ}

Рассмотрим эволюционное уравнение со случайными параметрами $\xi$

$$
u_{t}=A u+F(u, \xi)
$$


где $u(t)$ - состояние системы, лежащее в банаховом пространстве $H, A$ - некоторый, возможно неограниченный, линейный оператор, $F$ - нелинейный оператор, зависящий от случайного параметра $\xi$.

Системы подобного рода при естественных ограничениях на операторы $F$ и $A$ рассматривались в работах [9]-[11]. В частности, начально-краевые задачи для систем типа систем реакция-диффузия (химической кинетики) сводятся к уравнению (1) при подходящем выборе пространства $H$. При достаточно общих предположениях удается доказать, что уравнение (1) определяет глобальный полупоток (т. е. динамическую систему) [10]-[13], который может иметь конечномерные аттракторы [11]-[13], [9] (для случая, когда случайные параметры отсутствуют). При некоторых ограничениях на случайные параметры можно доказать существование решений уравнения (1) на полуоси $(0,+\infty)$.

Проблему гомеостазиса можно формализовать в рамках теории выживания [2]. Предположим, что система поддерживает гомеостазис, пока ее состояние $и$ остается внутри некоторой области $\Pi \subset H$ (область допустимых состояний). Численной характеристикой устойчивости гомеостазиса на интервале времени $[0, T]$ является вероятность $P\left(T, \Pi, u_{0}\right)$ того, что состояние системы останется в области П в течение интервала времени $[0, T]$, при условии, что в начальный момент система находилась в состоянии $u_{0}$. Гипотеза Громова-Карбоне утверждает, что

$$
P\left(T, \Pi, u_{0}\right) \rightarrow 0, \quad T \rightarrow+\infty .
$$

Мы доказываем асимптотику (2) для систем химической кинетики общего вида.

Далее мы рассматриваем так называемые гибридные динамические системы [14]. В них состояние $(u, s)$ задано с помощью двух типов переменных - спиновых $s=$ $\left(s_{1}, s_{2}, \ldots, s_{N}\right), s_{i} \in\{-1,1\}$, и непрерывных $u \in H$. Динамика переменной $u$ определена, как и выше, уравнением

$$
u_{t}=A u+F(u, \xi, s) .
$$

Динамика переменных $s$ может быть задана, например, случайным конечным автоматом, с вероятностями перехода, зависящими от $u, \xi$ [14]. Важные приложения такие модели находят, например, в биологии, где гены часто рассматриваются как булевы переменные (ген либо активирован, либо нет) [15]. В данной статье мы рассмотрим ситуацию, когда $s$ не зависит от времени. Стационарное решение $u_{0}$ в этом случае зависит от $s$, и нас интересует зависимость $s(T)$, дающая наименьшее значение вероятности $P_{\text {out }}=1-P\left(T, u_{0}(s), s\right)$ разрушения структуры при больших $T$. Ниже мы конкретизируем вид эволюционного уравнения.

\section{3. СИСТЕМЫ ХИМИЧЕСКОЙ КИНЕТИКИ СО СЛУЧАЙНЫМИ ПАРАМЕТРАМИ}

В физике, механике и биологии возникают уравнения химической кинетики (системы реакции-диффузии).

Рассмотрим следующие системы уравнений:

$$
\frac{\partial u_{i}}{\partial t}=d_{i} \Delta u_{i}+F_{i}(u, x, \xi(t))
$$


где

$$
F_{i}=f_{i}(u, x)+g_{i}(u, x) \cdot \xi(t), \quad g_{i}(u, x) \cdot \xi(t)=\sum_{k=1}^{p} g_{i}^{(k)}(u, x) \xi_{k}
$$

$u=\left(u_{1}, u_{2}, \ldots, u_{n}\right), u_{i}(x, t)$ - неизвестные функции, $x \in \Omega, t>0, i=1,2, \ldots, n$, $f_{i}(u, x), g_{i}^{(k)}(u, x) \in C^{2}\left(\mathbb{R}^{n} \times \Omega\right)$, ограниченная область $\Omega \subset \mathbb{R}^{m}$ имеет непустую внутренность и гладкую границу $\partial \Omega, d_{i}>0$ - коэффициенты диффузии. Пусть $\xi=\left(\xi_{1}, \ldots, \xi_{p}\right)$ - случайный процесс с значениями в $\mathbb{R}^{p}$ такой, что траектории этого процесса почти всегда кусочно-непрерывны. Мы ставим следующие граничные и начальные условия:

$$
\begin{gathered}
\frac{\partial u_{i}}{\partial \mathbf{n}}(x, t)=0, \quad x \in \partial \Omega, \quad t>0, \\
u_{i}(x, 0)=\phi_{i}(x), \quad\left|\phi_{i}\right|<C_{0},
\end{gathered}
$$

где $\mathbf{n}$ - вектор нормали к границе, функции $\phi_{i} \in C^{2}(\Omega)$.

Введем множество П допустимых состояний $u \in H$. Предположим, что $H \subset$ $L_{\infty}(\Omega)$ и

$$
\Pi=\{u \in H: \quad u(x) \in D \quad \forall x \in \Omega\}
$$

где $D$ - открытая ограниченная область в $\mathbb{R}^{n}$ с гладкой границей $\partial D$. Такой выбор П означает, что наша система разрушается, как только $u(x, t) \in \partial D$ для некоторых $x, t$. Следовательно, можно модифицировать функции $f, g^{(k)}$ для $u \notin D$, что упрощает доказательство существования решений системы уравнений (4) для всех $t>0$. Пусть $D \subset D^{\prime}$, где $D^{\prime}$ - открытое множество, и расстояние между границами областей $D^{\prime}, D$ не меньше некоторого $\delta>0$. Предположим, что вне $D^{\prime}$ векторные поля $f, g^{(k)}$ обращаются в нуль:

$$
f(u, x)=0, \quad g^{(k)}(u, x)=0, \quad u \notin D^{\prime}, \quad x \in \Omega .
$$

Относительно процесса $\xi$ мы предполагаем, что он является марковским и что выполняется следующее свойство. Обозначим через $V(\delta, z(\cdot))$ цилиндрическую окрестность траектории $t \rightarrow z(t)$ в $\mathbb{R}^{p}, t \in[0, T]: V=\left\{u \in \mathbb{R}^{p}\right.$, dist $\left.\{u, z(\cdot)\}<\delta\right\}$.

ПРЕДПОЛОЖЕНИЕ 1. Для любого $T$ и любой непрерывной траектории $t \rightarrow z(t)$ в $\mathbb{R}^{p}, t \in[0, T]$, и любого числа $\delta>0$ вероятность того, что существует траектория прочесса $\xi(t), t \in[0, T]$, полностью лежащая в иилиндрической окрестности $V(\delta, z(\cdot))$ траектории $z$, положительна:

$$
\operatorname{Prob}\{\xi: \xi(t) \in V(\delta, z(\cdot)), t \in[0, T]\}>\mu>0 .
$$

Данное предположение верно для многих случайных процессов. Вероятность попасть в окрестность $V$ может быть оценена с помощью теории больших уклонений [3]. 


\section{4. СУЩЕСТВОВАНИЕ И ЕДИНСТВЕННОСТЬ}

С помощью методов, изложенных в [10], можно доказать локальное по времени существование и единственность решений (для почти всех реализаций $\xi$ ) при подходящем выборе фазового пространства $H$. Фиксируем некоторую траекторию $\xi$. Мы представляем систему (4) в виде уравнения (1), вводя операторы $A$ и $F$ с помощью формул $(A u)_{i}=d_{i} \Delta u_{i}, F: u \rightarrow f(u, x)+g_{i}(u, x) \cdot \xi(t)$. В качестве $H$ берем прямое произведение $n$ пространств $L_{q}(\Omega)$ с $q>2 / m, m=\operatorname{Dim} \Omega$. Тогда оператор $A$ при подходящем выборе области определения становится секториальным оператором в пространстве $H$ [10]. Следовательно, этот оператор порождает некоторую полугруппу и пространства $H^{\alpha}, \alpha \in[0,1)$, состоящие из функций $u \in H$ с ограниченной нормой $\|u\|_{\alpha}=\left\|A^{\alpha} u\right\|_{L_{q}(\Omega)}$. Для доказательства локального существования решений системы (4) (на некотором открытом интервале времени) достаточно показать, что для некоторого $\alpha, 0<\alpha<1$, оператор $F$ ограничен, как оператор из $H^{\alpha}$ в $H$. Это является стандартным фактом, который доказывается с помощью теорем вложения [10].

Чтобы доказать существование решений для всех $t$, необходимо получить некоторые априорные оценки. Для рассматриваемых систем эти оценки можно получить с помощью известного принципа верхних и нижних решений [16].

Введем некоторые обозначения. На множестве векторов $v \in \mathbb{R}^{n}$ можно ввести отношение частичного порядка $v \leqslant w$, если $v_{i} \leqslant w_{i}$ для каждого $i$. Для $v \leqslant w$ обозначим через $E(v, w)$ множество векторов, лежащих между $v, w: E(v, w)=\{u \in$ $\left.\mathbb{R}^{n}, v \leqslant u \leqslant w\right\}$.

ПРИНЦИП ВЕРХНИХ И НИЖНИХ РЕШЕНИЙ ДЛЯ СИСТЕм. Pассмотрим cucmeму (4) при граничных условиях (6). Предположим, что имеются функции $U^{+}(t)$, $U^{-}(t)$, зависящие только от времени и такие, что

$$
\frac{d U_{i}^{+}}{d t}>\Phi_{i}^{+}(U), \quad \frac{d U_{i}^{-}}{d t}<\Phi_{i}^{-}(U),
$$

где

$$
\Phi_{i}^{+}=\max _{x} \max _{u \in E\left(U^{-}(t), U^{+}(t)\right)} F_{i}(u, x, t), \quad \Phi_{i}^{-}=\min _{x} \min _{u \in E\left(U^{-}(t), U^{+}(t)\right)} F_{i}(u, x, t) .
$$

Тогда если

$$
U_{i}^{+}(0)>\phi_{i}(x), \quad U_{i}^{-}(0)<\phi_{i}(x)
$$

для всех $x \in \Omega$, то решение системы (4) удовлетворяет неравенствам

$$
U^{-}(t) \leqslant u(x, t) \leqslant U^{+}(t), \quad x \in \Omega, \quad t>0 .
$$

Применим этот принцип. Рассмотрим произвольный интервал времени $[0, T]$ и с учетом (9) введем обозначения $C_{f}=\sup _{i, x \in \Omega, u \in D^{\prime}}\left|f_{i}(u, x)\right|, C_{g}=\sup _{i, k, u \in D^{\prime}}\left|g_{i k}(u)\right|$, $C_{0}(t)=\sup _{k}\left|\xi_{k}(t)\right|$. Положим $U_{i}^{-}=-R(t), U_{i}^{+}=R(t)$, где $R(t)$ определяется как решение дифференциального уравнения

$$
\frac{d R}{d t}=C_{f}+p C_{g} C_{0}(t), \quad R(0)>\sup _{x \in \Omega}|\phi(x)| .
$$


Неравенства (10) выполняются, следовательно, $\left|u_{i}\right|<R(t)$, что дает нужную априорную оценку, поскольку $R(t)$ ограничено на каждом ограниченном интервале. Тем самым глобальное существование решений системы (4) доказано для почти всех реализаций $\xi$.

\section{5. НЕУСТОЙЧИВОСТЬ СИСТЕМ С ФИКСИРОВАННЫМИ ПАРАМЕТРАМИ}

Используя результаты геометрической теории управления [17], мы докажем здесь теорему о неустойчивости (неизбежности выхода решения из области П). Мы предполагаем, что справедливо предположение 1.

Докажем две вспомогательные леммы. В них используется понятие общего положения, стандартное для дифференциальной топологии (см. [18]). Предположим, что $B_{0}, B_{1}$ - малые замкнутые шары ненулевого радиуса в $D^{\prime}, \operatorname{paccтояние~} \operatorname{dist}\left(B_{j}, D\right)$ которых до области $D$ положительно: $\operatorname{dist}\left(B_{j}, D\right)>\delta_{0}>0$, при этом $B_{0} \subset B_{1}$, $\operatorname{dist}\left(B_{0}, \partial B_{1}\right)>\delta_{1}>0$.

Лемма 1. Рассмотрим систему обыкновенных дифференииальных уравнений со случайными коэфбициентами

$$
\frac{d u_{i}}{d t}=f_{i}(u)+g_{i}(u) \cdot \xi, \quad u(0)=u_{0},
$$

в области $D^{\prime}$. Предположим, что векторное поле $f$ тождественно равно нулю в области $D^{\prime}, p \geqslant 2$ и векторные поля $g^{(k)} \in C^{\infty}\left(D^{\prime}\right)$ находятся в общем положении. Тогда справедливы следующие утверждения.

1. Существует конечное множество $Z$ непрерывных функиий $z^{(j)}(t), t \in\left[0, T_{j}\right]$, $j=1,2, \ldots, M$, обладающее следующим свойством. Для каждого $u_{0} \in D$ найдется $\xi \in Z$ такое, что соответствующая траектория $u\left(t, u_{0}, \xi(\cdot)\right)$ входит в $B_{1}$ :

$$
u\left(T_{l}, u_{0}, \xi(\cdot)\right) \in B_{1} .
$$

2. Существуют $T_{1}, \epsilon>0$ такие, что

$$
P\left(T_{1}, D, u_{0}\right)<1-\epsilon
$$

ДокАЗАтельство. Сначала отметим, что лемма неверна в случае $p=1$. Чтобы заметить это, достаточно рассмотреть одномерный случай, $u \in \mathbb{R}$, взять в качестве $D, D^{\prime}$ интервалы на вещественной оси и поле $g^{(1)}$, которое обращается в нуль в некоторой точке $u_{0}$ внутри $D$, при этом производная этого поля в этой точке не равна нулю. Тогда некоторые траектории (13) никогда не достигают границы $\partial D^{\prime}$. Все близкие к $g^{(1)}$ поля также порождают систему (13) с тем же свойством.

Доказательство леммы использует известный результат [17] геометрической теории управления, который основан на теореме трансверсальности Тома [18].

Рассмотрим функции $\xi_{k}$ как управления. Поставим задачу достижимости подмножества точек $B \subset D^{\prime}$, не лежащего в $D$, из начальной точки $u_{0}$ с помощью выбора $\xi_{k}(t), t \in\left[0, T_{0}\right][19]$. Векторные поля $g^{(k)}, k=1,2, \ldots, p$, порождают симметричную полидинамическую систему [17], [19]. Замыкание множества траекторий 
системы (13) содержит множество траекторий полидинамической системы (чтобы убедиться в этом, достаточно взять кусочно-постоянные управления $\left.\xi_{k}(t)\right)$. Симметричная полидинамическая система на многообразии, порожденная не менее чем двумя бесконечно гладкими векторными полями, находящимися в общем положении, обладает свойством полной управляемости. Это означает, что для любых двух точек имеется траектория, идущая из одной точки в другую [17]. Следовательно, для нашей системы и любой начальной точки $u_{0}$ имеется траектория, идущая из этой точки в $B$. Пусть эта траектория $u\left(t, u_{0}\right)$ порождена управлением $\xi(t)=z(t)$, $t \in\left[0, T_{0}\right]$.

Пусть $\tilde{u}_{0}-$ другое начальное условие, $\operatorname{dist}\left(u_{0}, \tilde{u}_{0}\right)<\kappa$. В силу теорем о непрерывной зависимости решений систем дифференциальных уравнений от начальных условий управление $z(t), t \in\left[0, T_{0}\right]$, будет порождать близкие траектории системы (13), которые войдут в $B_{1}$ в тот момент, когда исходная траектория $u\left(t, u_{0}\right)$ будет в $B$, если $\kappa$ достаточно мало.

Можно найти конечное множество $v_{1}, \ldots, v_{M}$ точек из $D$ таких, что их $\kappa$-окрестности покрывают все множество $D$. Имеются функции $z^{(j)}(t), t \in\left[0, T_{j}\right]$, такие, что соответствующие траектории $u\left(t, v_{j}, z^{(j)}(\cdot)\right)$ в момент $t=T_{j}$ лежат в $B$. Это доказывает первое утверждение леммы.

Моменты выхода $T_{j}$ могут быть выбраны одинаковыми, $T_{j}=T_{*}$. Это может быть доказано с помощью масштабного преобразования. Если управление $z(t)$, $t \in\left[0, T_{0}\right]$, приводит соответствующую траекторию в $B$, то управление $\tilde{z}(t)=a z(a t)$, $t \in\left[0, T_{0} / a\right]$, также выводит соответствующую траекторию в $B$. Тогда время выхода траектории системы (13) за границу области $D$ не превышает некоторой константы $T_{1}>0$, которая может быть выбрана не зависящей от начальной точки $u_{0}$.

Используя предположение 1, получаем утверждение 2 леммы.

Лемма 2. Рассмотрим систему (13) в области $D^{\prime}$. Предположим, что векторное поле $f \in C^{1}\left(D^{\prime}\right), p \geqslant 2$ и векторные поля $g^{(k)} \in C^{\infty}\left(D^{\prime}\right)$ находятся в общем положении. Тогда, при некоторых $T_{1}, \epsilon>0$ выполнено условие (15) для каждого $u_{0}$ $u$

$$
P\left(T, D, u_{0}\right) \rightarrow 0, \quad T \rightarrow \infty
$$

ДокАзАТЕльство. Сначала мы применим рассуждения, аналогичные используемым в доказательстве леммы 1 . Найдем управление $\xi=\tilde{z}(t), t \in\left[0, \widetilde{T}_{0}\right]$, которое приводит траекторию системы (13) за пределы области выживания. Основная идея состоит в том, что надо рассмотреть $\xi(t)$ большой амплитуды, но на малом интервале времени. В этом случае слагаемое $f$ несущественно.

Положим $f=0$. Используя лемму 1 , рассмотрим управление $\tilde{z}(t)=a z(a t)$, $t \in\left[0, T_{0} / a\right]$, которое выводит соответствующую траекторию $u_{a}$ системы $(13)$ в $B$ при $t=T_{0} / a$. Рассмотрим соответствующую траекторию $\tilde{u}_{a}(t)$ системы $(13)$ с ненулевым $f$. Сравним эти траектории, для этого введем функцию $w(t)=\tilde{u}_{a}-u_{a}$. Имеем

$$
\frac{d w}{d t}=f\left(\tilde{u}_{a}\right)+\left(g\left(\tilde{u}_{a}\right)-g\left(u_{a}\right)\right) \cdot z_{a}(t)
$$


Интегрируя по времени и вычисляя норму обеих частей, имеем оценку

$$
|w(t)| \leqslant C_{f} t+a C_{0} \int_{0}^{t}|w(s)| d s,
$$

где $C_{f}=\sup _{u \in D^{\prime}}|f|, C_{0}=c_{0} C_{g}, C_{g}=\sup _{u \in D^{\prime}}|D g|, c_{0}=\sup _{t \in\left[0, T_{0}\right]}|z(t)|$. Из оценки (17) с помощью неравенства Гронуолла [10] получаем

$$
|w(t)| \leqslant a^{-1} C_{f} T_{0} e^{a C_{0} t}, \quad t \in\left[0, \frac{T_{0}}{a}\right] .
$$

Подставляя в последнее неравенство $t=T_{0} / a$, имеем

$$
\left|w\left(\frac{T_{0}}{a}\right)\right| \leqslant C_{2} a^{-1}
$$

откуда для достаточно большого а следует, что траектория $\tilde{u}_{a}(t)$ системы $(13)$ с ненулевым $f$ покидает $D$ (в самом деле, точка $u_{a}\left(T_{0} / a\right)$ лежит на конечном расстоянии от $D$, не зависящем от $a$, а разность $w\left(T_{0} / a\right)=\tilde{u}_{a}-u_{a}$ может быть сделана сколь угодно малой за счет выбора $a$ ).

Согласно предположению 1 множество траекторий процесса $\xi$, лежащих в любой малой цилиндрической окрестности $\xi=z_{a}(t), t \in\left[0, T_{0} / a\right]$, имеет ненулевую меру. Отсюда заключаем, что существуют $T_{1}, \epsilon$ такие, что выполнено неравенство (15). Из этой оценки и марковского свойства процесса следует справедливость (16). Лемма доказана.

Теорема. Рассмотрим систему уравнений (4). Предположим, что

$$
\sup _{x \in \Omega, u \in D}\left|\frac{\partial f(u, x)}{\partial u}\right|<C
$$

и что поля $g^{(k)}(u, x) \in C^{\infty}(D) \times C^{3}(\Omega)$ для некоторого $x$ удовлетворяют всем условиям леммы 2, т.е. находятся в общем положении. Кроме того, пусть производная по нормали этих полей равна нулю на границе:

$$
D_{n} g^{(k)}(u, x)=0, \quad x \in \partial \Omega \quad \forall u .
$$

Тогда выполнена асимптотика (2), причем независимо от выбора началъного условия $\phi=u_{0}$.

ДокАЗАтЕЛЬство. Рассмотрим следующую систему обыкновенных дифференциальных уравнений с параметром $x$, ассоциированную с исходной системой (4):

$$
\frac{\partial w_{i}}{\partial t}=F_{i}(w, x, \xi(t))
$$

Здесь пространственная координата входит как параметр. Первая часть рассуждений повторяет доказательство лемм 1,2 . Пусть $u=u_{0}$ таково, что поля $g^{(k)}(u, x)$ находятся в общем положении. Рассмотрим управление $z_{a}(t)=a z(a t), t \in\left[0, T_{0} / a\right]$, которое выводит соответствующую траекторию $u_{a}\left(t, u_{0}\right)$ в $B$ для некоторого $T_{0} / a$. 
Соответствующее решение системы (18) обозначим через $w(x, t)$, а системы (4) через $u(x, t)$, опуская зависимость от параметра $a$.

Введем новую неизвестную функцию $v=u_{a}-w_{a}$. Для нее получаем систему уравнений

$$
\frac{\partial v_{i}}{\partial t}=d_{i} \Delta v_{i}+F_{i}(w, x, \xi(t))-F_{i}(u, x, \xi(t))+\rho_{i}(x, t, a),
$$

где $\rho_{i}(x, t, a)=d_{i} \Delta w_{i}$. Получим оценки для производных $\rho$ по $x$. Дифференцируя обе части (18) по $x$, имеем

$$
\left(D_{x} w\right)_{t}=D_{x} f(w, x)+D_{w} f(w, x) D_{x} w+a\left(g(w, x)_{x}+D_{w} g(w, x) D_{x} w\right) \cdot z(a t) .
$$

Интегрируя по времени это равенство, находим

$$
\begin{aligned}
\left(D_{x} w\right)(t)=D_{x} w(0)+\int_{0}^{t}\left(D_{x} f(w, x)+D_{w} f(w, x) D_{x} w+\right. \\
\left.\quad+a\left(D_{x} g(w, x)+D_{w} g(w, x) D_{x} w\right) \cdot z(a s)\right) d s, \quad t \leqslant \frac{T_{0}}{a} .
\end{aligned}
$$

Используя наши предположения о функциях $f$ и $g$, получаем интегральное неравенство

$$
\left|\left(D_{x} w\right)(t)\right| \leqslant c_{1}+c_{2} \int_{0}^{t}\left|D_{x} w(s)\right|(1+a|z(s a)|) d s, \quad t \leqslant \frac{T_{0}}{a},
$$

при этом, как следует из доказательств лемм $1,2,|z(s a)|<C_{0}$. Это неравенство дает оценку

$$
\left|D_{x} w(t)\right|<c_{3} e^{c_{4} a t}, \quad 0 \leqslant t \leqslant \frac{T_{0}}{a},
$$

где постоянные $c_{i}$ здесь и ниже не зависят от $a$. Итак,

$$
\left|D_{x} w(t)\right|<c_{5}, \quad 0 \leqslant t \leqslant \frac{T_{0}}{a} .
$$

Аналогично можно оценить вторые производные, дифференцируя систему (18) еще раз. В итоге получаем

$$
\left|\rho_{i}\right|<c_{6}, \quad 0 \leqslant t \leqslant \frac{T_{0}}{a} .
$$

Рассмотрим оценку функции $G=F_{i}(w, x, \xi(t))-F_{i}(u, x, \xi(t))$. Из явной формулы для $F_{i}$ и наших предположений вытекает, что

$$
\left|G_{i}\right| \leqslant c_{7} a|v|, \quad 0 \leqslant t \leqslant \frac{T_{0}}{a} .
$$

Теперь выберем верхнее и нижнее решения для системы (20) в виде

$$
V^{+}=\delta e^{c_{8} a t}, \quad V^{-}=-\delta e^{c_{8} a t},
$$

где $\delta>0$ - малая постоянная, не зависящая от $a, c_{8}>0$ - постоянная, не зависящая от $a$. Используя $(21)$ и $(20)$, можно проверить, что решения $V^{ \pm}$в самом деле являются верхним и нижним решениями при подходящем выборе $\delta, c_{8}$. Но тогда если $w\left(x, T_{0} / a\right) \in B$, то $u\left(x, T_{0} / a\right) \notin D$, поскольку $\operatorname{dist}(B, D)>r_{0}>0$ и, как следует из последней оценки, $\left|v\left(x, T_{0} / a\right)\right|<c_{9} \delta$. Мы можем выбрать $\delta<r_{0} / c_{9}$. Итак, мы доказали, что решение $u(x, t)$ покидает область П. Затем мы повторяем рассуждения из доказательства леммы 2. Теорема доказана. 
Сделаем несколько замечаний. Обобщение этих результатов на случай нелинейного шума (задача, имеющая большое физическое значение) выглядит не вполне тривиальным, поскольку теряется симметричность соответствующей полидинамической системы. Леммы 1, 2 не удается доказать тем же методом. Метод доказательства теоремы в этом случае тоже нуждается в модификации. Отметим, что даже задача локальной управляемости в случае несимметричной полидинамической системы нетривиальна [19]-[21].

Важно проследить, как вероятность выхода из области П зависит от коэффициентов диффузии, числа компонент и вида структуры. Чтобы сделать это, достаточно проанализировать оценку функции $\rho$ из доказательства теоремы. Получается, что наиболее неустойчивы пространственно однородные структуры в однокомпонентном случае, а наиболее устойчивы пространственно неоднородные (контрастные) структуры в многокомпонентном случае, когда часть коэффициентов диффузии мала (порядка $\epsilon$ ), а другая часть порядка единицы. Эти структуры хорошо изучены [22]. В разделе 6 мы рассмотрим нижнюю оценку вероятности $P\left(T, \Pi, u_{0}\right)$ для таких структур.

\section{6. ПЕРИОДИЧЕСКИЕ СТРУКТУРЫ С МАЛОЙ ВЕРОЯТНОСТЬЮ РАЗРУШЕНИЯ}

Сначала мы исследуем ситуацию, когда рассматривается динамика с непрерывным временем, заданная достаточно общими дифференциальными уравнениями в частных производных.

6.1. Некоторые предварительные оценки. Рассмотрим эволюционное уравнение

$$
\frac{d u}{d t}=A u+f(u, s)+g(u, s) \cdot \xi(t),
$$

где $A$ - отрицательно определенный самосопряженный оператор с областью определения $\operatorname{Dom}(A) \subset H$ и дискретным спектром, $H$ - гильбертово пространство, $u \in H$, $s=\left(s_{1}, s_{2}, \ldots, s_{N}\right), s_{i}(t) \in\{0,1\}, N=N(t)$ - натуральное число, для каждого $s$ отображения $f(\cdot, s), g(\cdot, s)-C^{2}$-отображения из некоторой (не зависящей от $s$ ) окрестности $W(R)=\left\{u:\|u\|_{\alpha}<R\right\}$ нуля в $H^{\alpha}, \alpha \in(0,1)$, где $R>0$. Обозначим через $\sigma$ число, ограничивающее спектр $A$ слева:

$$
\operatorname{Spec} A<-\sigma, \quad \sigma>0 .
$$

Пусть $\psi_{n}$ - собственные функции оператора $A: A \psi_{n}=-\lambda_{n} \psi_{n}, \lambda_{n} \rightarrow \infty$ при $n \rightarrow \infty$.

Предположим, что для каждого $s$ имеется стационарное решение $\bar{u}(s)$ детерминированной части эволюционного уравнения: $A \bar{u}(s)+f(\bar{u}(s), s)$. Предположим, кроме того, что П - открытое подмножество окрестности $W(R)$, содержащее все $\bar{u}(s)$ вместе с их малыми окрестностями. Пусть

$$
\|f(u, s)-f(\bar{u}(s), s)\|<C_{0}\|u-\bar{u}(s)\|_{\alpha}^{2}, \quad C_{0}>0,
$$

постоянная $C_{0}$ не зависит от $s$ и $u$.

Относительно $\xi$ сделаем следующее предположение. 
ПРЕДПОЛОЖЕНИЕ 2. Введем функиионалы случайного процесса

$$
\phi_{T}(\xi(\cdot))=\sup _{t \in[0, T]}|\xi(t)|
$$

где $\alpha \in(0,1)$. Предположим, что

$$
\mathrm{P}\left\{\xi: \phi_{T}(\xi(\cdot))>a\right\} \rightarrow 0, \quad a \rightarrow \infty
$$

Данный функционал хорошо изучен [3].

Напомним, что оператор $A$ порождает полугруппу $e^{A t}$ и выполнены оценки [10], которые следуют из разложения по собственным функциям:

$$
\begin{aligned}
\left\|e^{A t} u\right\|_{\alpha} & \leqslant\|u\|_{\alpha} e^{-\sigma t} \\
\left\|e^{A t} u\right\|_{\alpha} & \leqslant c_{\alpha} \max \left\{1, t^{-\alpha}\right\}\|u\| e^{-\sigma t} .
\end{aligned}
$$

Пусть $\mathcal{P}_{n}-$ проектор на подпространство $H^{n}=\operatorname{Span}\left\{\psi_{1}, \psi_{2}, \ldots, \psi_{n}\right\}$, натянутое на первые $n$ собственных функций оператора. Обозначим через $\mathcal{Q}_{n}=I-\mathcal{P}_{n}$ проектор на подпространство, ортогональное $H^{n}$. Имеются оценки, аналогичные (26):

$$
\begin{gathered}
\left\|e^{A_{n} t} u\right\|_{\alpha} \leqslant\|u\|_{\alpha} e^{-\lambda_{n} t}, \\
\left\|e^{A_{n} t} u\right\|_{\alpha} \leqslant c_{\alpha, n} \max \left\{1, t^{-\alpha}\right\}\|u\| e^{-\lambda_{n} t}, \quad A_{n}=\mathcal{Q}_{n} A .
\end{gathered}
$$

Следуя [10], [11], положим $u=\bar{u}+v+w, v=\mathcal{P}_{n}(u-\bar{u})$. Тогда

$$
\begin{aligned}
& \frac{d v}{d t}=\mathcal{P}_{n} A v+\mathcal{P}_{n}(\tilde{f}(v+w, s)+g(v+w, s) \cdot \xi(t)), \\
& \frac{d w}{d t}=A_{n} w+\mathcal{Q}_{n}(\tilde{f}(v+w, s)+g(\bar{u}+v+w, s) \cdot \xi(t)),
\end{aligned}
$$

где $\tilde{f}=f(u)-f(\bar{u})$. Рассмотрим эти уравнения на интервале $[0, T]$ и запишем их в интегральной форме:

$$
\begin{aligned}
v(t)= & e^{\mathcal{P}_{n} A t} v(0)+\int_{0}^{t} e^{\mathcal{P}_{n} A(t-\tau)} \mathcal{P}_{n}(\tilde{f}(v(\tau)+w(\tau), s)+g(\bar{u}+v(\tau)+w(\tau), s) \cdot \xi(\tau)) d \tau \\
w(t)= & e^{A_{n} t} v(0)+\int_{0}^{t} e^{A_{n}(t-\tau)} \mathcal{Q}_{n}(\tilde{f}(v(\tau)+w(\tau), s)+ \\
& +g(\bar{u}+v(\tau)+w(\tau), s) \cdot \xi(\tau)) d \tau, \quad t \geqslant 0
\end{aligned}
$$

Получим предварительную оценку решений (29). Предположим, что для некоторых $C_{1}>1,0<r<r_{0}$ на интервале $[0, t)$

$$
\|u(t)\|_{\alpha}<C_{1} r, \quad\|u(0)\|<r
$$

Обозначим

$$
G(r)=\sup _{v \in W(r)}\|g(\bar{u}+v, s)\|, \quad p_{n}(r)=\sup _{v \in W(r)}\left\|\mathcal{P}_{n} g(\bar{u}+v, s)\right\|
$$


Применяя оценки (26), (27) и следуя [10], получаем

$$
\begin{gathered}
\|v(t)\|_{\alpha} \leqslant e^{-\sigma t} r+c \int_{0}^{t} \max \left\{1,(t-\tau)^{-\alpha}\right\} e^{-\sigma(t-\tau)}(\|\tilde{f}(v(\tau)+w(\tau), s)\|+ \\
\left.+\left\|\mathcal{P}_{n} g(\bar{u}+v+w, s)\right\||\xi(\tau)|\right) d \tau, \quad t>t_{0}, \\
\|w(t)\|_{\alpha} \leqslant e^{-\lambda_{n} t} r+c \int_{0}^{t} \max \left\{1,(t-\tau)^{-\alpha}\right\} e^{-\lambda_{n}(t-\tau)}(\|\tilde{f}(v(\tau)+w(\tau), s)\|+ \\
\left.+\sup _{u \in W(R)}\|g(u, s)\||\xi(\tau)|\right) d \tau, \quad t>0 .
\end{gathered}
$$

Используя определение $\phi_{T}$, имеем при $t \in(0, T]$

$$
\int_{0}^{t} \max \left\{1,(t-\tau)^{-\alpha}\right\} e^{-\lambda_{n}(t-\tau)}|\xi(\tau)| d \tau<C(\alpha) \lambda_{n}^{\alpha-1} \phi_{T}(\xi(\cdot)) .
$$

Аналогично оцениваем и другие вклады в правые части (29). Это дает

$$
\begin{array}{clrl}
\|w(t)\|_{\alpha} \leqslant e^{-\lambda_{n} t} r+c_{3} r^{2}+c_{4} \lambda_{n}^{\alpha-1} G\left(C_{1} r\right) \phi_{T}(\xi(\cdot)), & & t>t_{0}, \\
\|v(t)\|_{\alpha} \leqslant e^{-\sigma t} r+c_{3} r^{2}+c_{5} p_{n}\left(C_{1} r\right) \phi_{T}(\xi(\cdot)), & & t>0 .
\end{array}
$$

С помощью этих оценок можно доказать вспомогательную лемму.

ЛЕмма 3. Предположим, что переменные s фиксированы. При условиях

$$
\begin{aligned}
c_{4} p_{n}(2 r) \phi_{T}(\xi(\cdot)) & <\frac{r}{8}, \\
c_{5} G\left(C_{1} r\right) \lambda_{n}^{1-\alpha} \phi_{T}(\xi(\cdot)) & <\frac{r}{8}, \\
4 c_{3} r & <1,
\end{aligned}
$$

если решение и в момент $t_{0}$ лежит в $W(r)$, оно существует, единственно и лежит в $W(2 r)$ для всех $t \in(0, T]$.

ДокАЗАТЕЛьство основано на стандартных оценках [10]. Для каждого $t>t_{0}$ рассмотрим правые части $(29)$ как оператор $S_{t}: u(\cdot) \rightarrow S_{t}(u)$, определенный на шаре $W(2 r)$. Локальное (по времени) существование и единственность доказываются методом, изложенным в монографии [10]. При помощи оценок для полугрупп (26), (27) можно проверить [10], что $S_{t}$ является сжимающим оператором при малых $t-t_{0}$. Глобальное существование следует из этих оценок и неравенств (34), которые показывают, что оператор $S_{t}$ при всех $t>t_{0}$ отображает шар $W(2 r)$ в себя.

ЛЕмма 4. Предположим, что переменные s фиксированы. При условиях (35), (36) $u$

$$
2 e^{-\sigma \tau_{0}}<1
$$

если решение и в момент $t_{0}$ лежит в $W(r)$, оно лежит в $W(r)$ для всех $t=t_{0}+\tau$, где $\tau>\tau_{0}$.

ДокАЗАТЕЛЬСтво вытекает из предыдущей леммы и оценок (34). 
6.2. Оценки вероятности разрушения периодических структур. Применим леммы 3,4 . Предположим, что область П содержит малый шар $W\left(2 r_{0}\right)$. Выберем малые $r<r_{0}$, предположим, что они удовлетворяют условиям лемм 3,4 . Число $n$ будет подобрано ниже.

Обозначим через $P_{\text {оut }}(r, T)$ вероятность выхода $u(t)$ за пределы шара $W(2 r)$ при $0 \leqslant t<T$ или выхода $u(t)$ из $W(r)$ при $t=T$, при условии, что $u(0) \in W(r)$.

С помощью лемм 3,4 мы получаем оценку вероятности $P_{\text {out }}(r, T)$ :

$$
P_{\text {out }}(r, T)<\mathrm{P}\left\{\xi: \phi_{\alpha}(\xi(\cdot))>R_{n}\right\}
$$

где

$$
R_{n}=C_{1} r \min \left\{p_{n}^{-1}, G\left(C_{1} r\right)^{-1} \lambda_{n}^{1-\alpha}\right\} .
$$

Заметим, что $\lambda_{n} \rightarrow \infty$ при $n \rightarrow \infty$ и $\lambda_{n}$ не зависят от $s$. Чем больше $R_{n}$, тем меньше вероятность выхода. Чтобы получить максимальное $R_{n}$, мы должны уменьшить за счет выбора $s, N$ величину

$$
p_{n}(s)=\sup _{v \in W(r)}\left\|\mathcal{P}_{n} g(\bar{u}(s)+v, s)\right\| .
$$

Оказывается, что эта задача минимизации нетривиальна даже для простейшего случая, когда $g$ зависит от булевых переменных $s_{m}$ аддитивно:

$$
g(u, s)=\sum_{m=1} h_{m}(u) s_{m}
$$

Рассмотрим этот случай. Для каждого $n$ мы ищем $s=\left(s_{1}, s_{2}, \ldots, s_{N}\right)$, удовлетворяющие условию

$$
H_{n}(s, r)=\sup _{v \in W(r)}\left\|\mathcal{P}_{n} g(\bar{u}(s)+v, s)\right\|^{2}<\epsilon_{n}
$$

где $\epsilon_{n}>0$ таковы, что $\epsilon_{n} \rightarrow 0, n \rightarrow+\infty$.

Параметр $r$ в (43) может быть взят малым. Пренебрегая членами порядка $r$ и используя (42), получаем из последнего соотношения $H_{n}(s, r)=H_{n}(s)+O(r)$, где

$$
\begin{gathered}
H_{n}(s)=\sum_{m=1}^{N} \sum_{m^{\prime}=1}^{N} W_{m m^{\prime}}^{(n)} s_{m} s_{m^{\prime}} \\
W_{m m^{\prime}}^{(n)}=\left\langle\mathcal{P}_{n} h_{m}, \mathcal{P}_{n} h_{m^{\prime}}\right\rangle
\end{gathered}
$$

$\langle f, g\rangle$ - скалярное произведение в $L_{2}(\Omega)$.

Итак, мы пришли к энергии $H_{n}(s)$ вида (44). Это фундаментальный гамильтониан, возникающий в теории спиновых стекол [23] и нейронных сетей [24]. При исследовании этого гамильтониана применялись сначала приближенные эвристические методы (строгие результаты см. в [6]). При специальном выборе $W_{m m^{\prime}}=$ $\sum_{l=1}^{p} K_{m l} K_{m^{\prime} l}, p \ll N$, гамильтониан $H$ называют гамильтонианом Хопфилда [24], хотя в действительности он впервые был рассмотрен Фиготиным и Пастуром [25]. Именно такая ситуация возникает в нашем случае, если мы выбираем $n \ll N$. Тогда, разлагая проектор по собственным функциям, получаем $K_{m l}=\left\langle h_{m}(\bar{u}), \psi_{l}\right\rangle$. 
Известно, что в общем случае задача минимизации гамильтониана $H_{n}(s) \mathrm{NP}$-трудная [26], т.е. неизвестен общий алгоритм, который во всех случаях за полиномиальное число $\operatorname{Poly}(N)$ шагов находит минимум $H_{n}$. Отметим также, что если мы обобщим формулу (42) и рассмотрим более сложные зависимости $g$ от $s$, мы можем получить более общие задачи булевой оптимизации, например задачу K-SAT [26]. В последнее время наметился определенный прогресс в понимании свойств таких задач [5], [7], [8] и в проблеме Хопфилда [24]. Показано, что для некоторых NP-трудных задач при определенных условиях на параметры задача со случайными параметрами разрешима за полиномиальное время. В нашем случае такая ситуация тоже возможна. Условие возможности нахождения конфигурации $s$, удовлетворяющей (43) в полиномиальное время Poly $(N)$, является естественным - в противном случае процесс нахождения структуры требовал бы слишком много времени. Кроме того, нам нет необходимости искать точный минимум гамильтониана, достаточно найти хорошую оценку сверху минимума.

6.3. Пример. Пусть $\Omega=[0, L], x \in \mathbb{R}$. Рассмотрим систему химической кинетики из раздела 5 на этом интервале с нулевыми условиями Неймана. Предположим, что в процессе эволюции с ростом $N$ происходит образование все более сложных одномерных слоистых структур. Такие стационарные периодические решения $\bar{u}(x, s)$ были впервые получены для систем химической кинетики в основополагающей работе Тьюринга [27]. Структуры, близкие к периодическим, наблюдаются в развитии эмбриона [4], [28]. Можно рассмотреть следующую ситуацию.

Пусть слой периодической структуры с номером $i$ имеет носитель $\Omega_{i}=[(i-$ 1) $b, i b], i=1,2, \ldots, N, b=L / N$. Предположим, что каждый ген контролирует реакцию системы на флуктуации только в соответствующем слое, это означает, что носитель $\operatorname{supp} h_{m}\left(\bar{u}_{m}(x, s)\right)=\Omega_{m}$. Тогда, если оператор есть $d^{2} / d x^{2}$, то оценка $K_{l m}$ дает $\left|K_{l m}\right|<c L^{1 / 2} N^{-1}$, поскольку собственные функции $\psi_{l}$ удовлетворяют неравенству $\left|\psi_{l}\right|<c L^{-1 / 2}$, где $c$ - различные константы, не зависящие от $L, N, n$. Следовательно, $\left|W_{m m^{\prime}}\right|<c \operatorname{Ln} N^{-2}$.

Рассмотрим гамильтониан $H_{n}$ как случайную функцию независимых случайных переменных $s_{i}$, принимающих значения $-1,1$ с вероятностями $1 / 2$. Оценка математического ожидания $H_{n}$ дает $E H_{n}=\sum_{m=1}^{N} W_{m m} s_{m}^{2}<c n L N^{-1}$. Применяя неравенство Маркова, имеем

$$
\operatorname{Prob}\left\{H_{n}>a\right\}<c_{0} n L N^{-1} a^{-1} \text {. }
$$

Положим $a=c_{0} n L(4 N)^{-1}$, тогда существует набор $s_{i}^{*}$ такой, что $H_{n}\left(s^{*}\right) \leqslant a(n, N, L)$ и он может быть найден методом случайного поиска, поскольку вероятность его найти за один шаг не менее $3 / 4$. Для $p_{n}$ получаем теперь оценку $p_{n}<a=c_{0} n L N^{-1}$. Далее заменяем $p_{n}$ его верхней оценкой, а для $G$ используем грубую оценку $G<C$.

Обратимся к выражению (40) для $R_{n}$. Число собственных функций $n(N, L)$ естественно выбрать с помощью соотношения $a^{-1}(n, N, L)=\lambda_{n}^{1-\alpha}$. Заметим, что $\lambda_{n}=c n^{2} L^{-2}$. Это дает $n=n_{*}(L, N)=\left(L^{1-2 \alpha} N\right)^{(3-2 \alpha)^{-1}}$. Следовательно,

$$
\Lambda(N, L)=R_{n^{*}}=c N^{\frac{2(1-\alpha)}{3-2 \alpha}} L^{\frac{4(\alpha-1)}{3-2 \alpha}} .
$$


Поскольку наша задача одномерная, согласно теоремам вложения можно взять любое $\alpha \in(1 / 2,1)$. Устойчивость структуры с $N$ слоями тем выше, чем больше $\Lambda$, она растет вместе с числом слоев и понижается с ростом размера $L$. Используя теперь оценку (39) и леммы 3,4 , получаем

ПреДЛОЖЕНИЕ. При сделанных выше предположениях вероятность $P_{\text {out }}(r, T)$ удовлетворяет неравенству

$$
P_{\text {out }}(r, T) \leqslant \operatorname{Prob}\left\{\phi_{T}(\xi(\cdot))>c \Lambda(N, L)\right\}, \quad c>0 .
$$

Тем самым, согласно (40) при $N \rightarrow \infty$ вероятность $P\left(T, u_{0}(s), s\right) \rightarrow 1$. Сходимость зависит, в частности, от свойств случайного процесса $\xi$. Если, например, процесс стандартный винеровский, то $\ln \operatorname{Prob}\left(\phi_{T}>\Lambda\right)<-c \Lambda^{2} T^{-1}$ при $\Lambda \rightarrow \infty$.

\section{7. ЗАКЛЮЧЕНИЕ}

Мы рассмотрели гипотезу о разрушении диссипативных структур под действием флуктуаций, сформулированную в интуитивной форме Громовым и Карбоне [1]. Мы предложили формализацию гипотезы и обнаружили, что в системах химической кинетики действительно наблюдается такой механизм разрушения структур, но при условии, что допускаются флуктуации сколь угодно большой амплитуды. Кроме того, анализ оценок из доказательства теоремы показывает, что более устойчивыми являются структуры, возникающие в многокомпонентных системах, где различные компоненты диффундируют с различными скоростями. Такие структуры часто встречаются в биологии, физике и механике и хорошо изучены [22]. В этих приложениях возникают слоистые периодические структуры.

Далее был рассмотрен случай, когда период и число слоев зависит от выбора некоторых дискретных переменных $s$ (в биологии это гены). Мы рассмотрели задачу поиска значений $s$, для которых вероятность разрушения структуры мала: $P\left(T, \Pi, u_{0}(s)\right)>1-\nu(N), \nu \rightarrow 0$ при $N \rightarrow \infty$, где $N$ - число слоев в структуре. Эта задача в простейшем случае сводится к задаче поиска $s$, дающих "хорошую" верхнюю границу энергии для гамильтониана Хопфилда. Удается показать, что такие $s$ могут быть найдены за полиномиальное время Poly $(N)$.

Благодарности. Мы благодарим проф. А. Л. Фрадкова (ИПМАШ РАН), проф. Н. Н. Петрова (СПбГУ) и проф. О. Радулеску (University of Montpelier) за полезные замечания и помощь. Работа поддержана РФФИ (гранты № 10-01-00627_а, 10-01-00814_а) и CDRF NIH (грант RR07801).

\section{Список литературы}

[1] M. Gromov, A. Carbone, Gaz. Math., 88, suppl. (2001), 80 pp.; Preprint IHES/M/01/03, 2001.

[2] J.-P. Aubin, Viability Theory, Systems and Control: Foundations and Applications, Birkhäuser, Boston, 1991.

[3] A. D. Ventsel, M. I. Freidlin, Random Perturbations of Dynamic Systems, Springer, New York, 1984.

[4] S. Vakulenko, M. Reinitz, J. Reinitz, O. Radulescu, Phys. Rev. Lett., 103:16 (2009), 168102, $4 \mathrm{pp}$. 
[5] M. Mézard, R. Zecchina, Phys. Rev. E, 66:5 (2002), 056126, 27 pp., arXiv: cond-mat/0207194.

[6] M. Talagrand, Spin Glasses: A Challenge for Mathematicians. Cavity and Mean Field Models, Ser. Modern Surv. Math., 46, Springer, Berlin, 2003.

[7] E. Friedgut, J. Amer. Math. Soc., 12:4 (1999), 1017-1054.

[8] D. Achlioptas, Theoret. Comput. Sci., 265:1-2 (2001), 159-185.

[9] R. Temam, Infinite-Dimensional Dynamical System in Mechanics and Physics, Appl. Math. Sci., 68, Springer, New York, 1997.

[10] D. Henry, Geometric Theory of Semilinear Parabolic Equations, Lecture Notes in Math., 840, Springer, Berlin, 1981.

[11] О. А. Ладыженская, УМН, 42:6(258) (1987), 25-60.

[12] Ю. С. Илященко, Успехи механики, 1 (1982), 31-63.

[13] J. K. Hale, Asymptotic Behavior of Dissipative Systems, Math. Surv. Monogr., 25, AMS, Providence, RI, 1988.

[14] A.V. Savkin, R. J. Evans, Hybrid Dynamical Systems, Controller and Sensor Switching Problems, Control Eng., Birkhäuser, Boston, MA, 2002.

[15] D. Thieffry, R. Thomas, Bull. Math. Biol., 57 (1995), 277-297.

[16] J. Smoller, Shock Waves and Reaction-Diffusion Equations, Springer, New York, 1983.

[17] C. Lobry, Chech. Math. J., 22(97) (1972), 230-237.

[18] М. Хирш, Дифференциальная топология, Мир, М., 1979.

[19] H. J. Sussmann, SIAM J. Control Optim., 25:1 (1987), 158-194.

[20] А.А. Аграчев, Ю.Л. Сачков, Геометрическая теория управления, Физматлит, М., 2005.

[21] Н. Н. Петров, Дифферени. уравнения, 4 (1968), 1218-1232.

[22] А.Б. Васильева, В.Ф. Бутузов, Н.Н. Нефедов, Фундамент. и прикл. матем., 4:3 (1998), 799-851.

[23] В. С. Доценко, УФН, 163:6 (1993), 1-37.

[24] J. J. Hopfield, Proc. Natl. Acad. Sci. USA, 79:8 (1982), 2554-2558.

[25] Л. А. Пастур, А. Л. Фиготин, ТМФ, 35:2 (1978), 193-210.

[26] T.H. Cormen, C. E. Leiserson, R. L. Rivest, C. Stein, Introduction to Algorithms, MIT Press, Cambridge, MA, 2001.

[27] A. M. Turing, Phil. Trans. R. Soc. Lond. Ser. B, 237:641 (1952), 37-72.

[28] E. Mjolsness, D. H. Sharp, J. Reinitz, J. Theoret. Biol., 152:4 (1991), 429-453.

Поступила в редакцию 18.01.2010, после доработки 13.04.2010 